\title{
Longitudinal analysis of the preterm cortex using multi-modal spectral matching
}

\author{
Eliza Orasanu ${ }^{1}$, Pierre-Louis Bazin ${ }^{2}$, Andrew Melbourne ${ }^{1}$, Marco Lorenzi ${ }^{1}$, \\ Herve Lombaert ${ }^{3}$, Nicola J. Robertson ${ }^{4}$, Giles Kendall ${ }^{4}$, Nikolaus Weiskopf ${ }^{2}$, \\ Neil Marlow ${ }^{4}$, and Sebastien Ourselin ${ }^{1}$ \\ ${ }^{1}$ Centre for Medical Image Computing, University College London, UK \\ ${ }^{2}$ Department of Neurophysics, Max Planck Institute for Human Cognitive and Brain \\ Sciences, Leipzig, Germany \\ ${ }^{3}$ INRIA - Microsoft Research Joint Centre, Palaiseau, France \\ ${ }^{4}$ Academic Neonatology, EGA UCL Institute for Women's Health, London, UK
}

\begin{abstract}
Extremely preterm birth (less than 32 weeks completed gestation) overlaps with a period of rapid brain growth and development. Investigating longitudinal brain changes over the preterm period in these infants may allow the development of biomarkers for predicting neurological outcome. In this paper we investigate longitudinal changes in cortical thickness, cortical fractional anisotropy and cortical mean diffusivity in a groupwise space obtained using a novel multi-modal spectral matching technique. The novelty of this method consists in its ability to register surfaces with very little shape complexity, like in the case of the early developmental stages of preterm infants, by also taking into account their underlying biology. A multi-modal method also allows us to investigate interdependencies between the parameters. Such tools have great potential in investigating in depth the regions affected by preterm birth and how they relate to each other.
\end{abstract}

\section{Introduction}

Infants born extremely preterm are at high risk of developing cognitive and neurologic impairment from an early age [1]. During the last trimester of pregnancy, the fetal brain undergoes several changes in size, shape, volume, appearance [2], as well as changes in connectivity and microstructure. Premature birth implies that this development of the infant brain will take place under the harsh conditions of the extra-uterine environment. Accurate measurements of the preterm brain during this early post-natal period may yield predictive biomarkers of neurological outcome. Furthermore, connecting information given by different imaging modalities (structural and diffusion), may begin to provide an understanding on the brain development during the preterm period and how it is affected by preterm birth.

Longitudinal studies are critical to the accurate analysis of neurodevelopment due to the rapidity of changes in shape and structure. However, longitudinal studies of this period of development are challenging for several reasons. 
First, the early and more developed infant brains have very different appearance and connectivity. Spectral techniques have been used in the preterm populations for matching white matter surfaces for the study of longitudinal cortical folding patterns and changes in the white-grey matter boundary [3]. These techniques have proved successful in the intra-subject case due to the prominance of primary sulci even at early gestational age allowing matching to be driven by low-frequency spatial features. Second, the anatomical variability of cortical surfaces is very high cross-sectionally, and variability is not well-represented by geometric folding patterns. This is especially true in the very early stages of development, when we deal with lissencephalic brains from different subjects. During the very-preterm period, additional diffusion information of the white matter might guide surface registration by contributing emergent microstructural information; high cortical fractional anisotropy (FA) is a feature of early brain development, although FA falls rapidly in the third trimester with cortical maturation. Matching of diffusion tensor images alone [4] to study longitudinal changes in diffusion parameters and white matter tract shape has already shown to be successful, but is of limited use to align cortical surfaces except within a very narrow age range.

The relationships between cerebral microstructure and cortical shape are intrinsically related and the ability to accurately combine multi-modal information about cortical and white matter structure with cortical shape information represents a key challenge to understanding the synergistic processes of neurodevelopment. In this paper we propose a novel registration technique based on Pairing Images using the Multi-Modal Spectra (PIMMS) to register crosssectional data from 9 early-scanned preterm infants and investigate longitudinal rates of change in cortical thickness, cortical FA and $\mathrm{MD}$, in the created groupwise space. The method matches the combined spectra based on tensor similarity (from the diffusion weighted images) and on the surface information (obtained from structural image segmentation). The proposed method has an advantage over the classical surface registration algorithm, since it optimises both surface and microstructural information, thus providing a more biologically accurate mapping based on tissue properties and not only sulcal patterns. The mapping also enables us to study multi-modal variations and interdependency between parameters obtained from different imaging modalities.

\section{Data and image processing}

Subjects Volumetric $\mathrm{T}_{1}$-weighted images were acquired for nine infants (Mean Gestational Age at Birth (GAB) of $26.8 \pm 1.5$ weeks) on a Philips Achieva 3T MRI machine. The infants were imaged at first shortly after birth, at a mean age of $26.8 \pm 1.1$ weeks equivalent gestational age (EGA) and then at a mean age of $41.7 \pm 2.9$ weeks EGA, in an MR-compatible incubator after feeding, when spontaneously asleep, with no sedation. $\mathrm{T}_{1}$-weighted data was acquired at a resolution of $0.82 \mathrm{~mm} \times 0.82 \mathrm{~mm} \times 0.5 \mathrm{~mm}$ at $\mathrm{TR} / \mathrm{TE}=17 / 4.6 \mathrm{~ms}$, acquisition duration $462 \mathrm{~s}$. The diffusion weighted data had a resolution of $1.75 \mathrm{~mm} \times 1.75 \mathrm{~mm} \times 2 \mathrm{~mm}$. 
We acquired six volumes at $b=0 \mathrm{~mm}^{2} / \mathrm{s}, 16$ directions at $b=750 \mathrm{~mm}^{2} / \mathrm{s}$ and 32 at $b=2000 \mathrm{~mm}^{2} / \mathrm{s}$ with $\mathrm{TR} / \mathrm{TE}=9 \mathrm{~s} / 60 \mathrm{~ms}$.

Image preprocessing and infant brain segmentation The preprocessing of the T1-weighted images was done as described in [5]. Briefly, images were bias-corrected then segmented using the help of neonate brain atlases and an adaptive EM algorithm. The preprocessing of the diffusion images was done as described in [6]. We obtained FA and MD maps by fitting a diffusion tensor model to the data. We resampled the diffusion images to $\mathrm{T} 1$ space using a rigid registration and correct for EPI distortions.

Cortical Thickness Using the obtained white matter, grey matter and CSF segmentations, we automatically computed the level set functions of the inner (WM/GM), central and outer (GM/CSF) boundaries of each hemisphere of the cerebral cortex using CRUISE [7]. We estimated the cortical thickness (CT) as the difference between the distance to the inner cortical surface and the distance to the outer cortical surfaces (given by the level set values). The WM/GM boundary level set was used to create smooth triangle based meshes of each hemisphere. We mapped the CT values onto the white-grey matter inner surface.

Laminar Analysis From the level set functions of the WM/GM and GM/CSF boundaries, we created a continuous layering of the cortex (cortical grey matter) [8]. We used the obtained 11 laminar profiles to sample the FA and MD maps, and computed the mean values of these parameters across the cortex. To reduce partial volume effects, we excluded the first and last three profiles from the computation of the average cortical FA and MD values. These mean FA and MD values were then mapped onto the white-grey surface.

Longitudinal and cross-sectional mapping To quantify the longitudinal changes taking place over the preterm period, we defined a mapping for the intra-subject WM/GM surfaces, by hemisphere, at the two timepoints, using JSM [9], initialised with a rigid CPD [10]. To investigate the changes in the same reference space, we create an average early time point template, by choosing a random subject as template, mapping all the others into its space and averaging the results. To register this WM/GM surface data, using only a surface-based matching technique based on mapping sulcal patterns would not be appropriate, since early cross-sectional data of this type does not provide us with sufficient surface information for a proper match. Hence we propose a novel multi-modal registration technique based on spectral matching (PIMMS), described in the following section.

\section{Pairing Images using Multi-Modal Spectra (PIMMS)}

To estimate a more biologically accurate surface matching in the case of lissencephalic surfaces, we propose the novel PIMMS, which uses both diffusion tensor images and surface information. Combining surface (2D) and volume (3D) information is not trivial [11]. We tackled this problem by embedding the surface with a level set representation in the 3D image domain, and reformulating the surface 
spectral matching problem in this context. We followed the previous strategies of spectral decomposition in the case of surfaces and diffusion tensor images. We then compared the groupwise average of PIMMS with the results of JSM.

Spectral Components of Surface in Image Domain To decompose the cortical surface, but in image space, we used the level set images of the white-grey matter boundary, $I_{L S}$. To optimise our decomposition, we considered a subset of our image, $I_{L S \Omega_{1}}$, consisting of the voxels around the boundary within a chosen threshold. Similarly to the surface decomposition, where we need to have continuous surfaces with no holes to obtain smooth spectra, we chose the smallest threshold that ensured a continuous surface for all subjects, which was found to be of $3.5 \mathrm{~mm}$ in the presented work.

We constructed the connected graph $(V, E)$ with the vertices $V$ being image voxels and the edges $E$ are defined by the neighbourhood structure of these vertices. We then represented the graph with its adjacency matrix $W$, where for each pair of voxels $x_{i}$ and $x_{j}, x_{i} \neq x_{j}, W_{i j}$ is 1 if the voxels are neighbours and 0 if they are not. The diagonal matrix $D$ gives the total weighting of all edges connected to each voxel and is computed by $D_{i i}=\sum_{j} W_{i j}$. The general graph Laplacian is defined by $\mathcal{L}=G^{-1}(D-W)$, with $\mathrm{G}$ being the diagonal node weighting matrix, which we computed according to the each voxel i's inverse level set value $G_{i i}=1 / x_{i}$. Hence, elements closer to the boundary, with a smaller level set value, will have a higher weighting when computing the spectra.

The graph spectrum of the level set image at the defined points is given by the eigen-decomposition of the general graph Laplacian $\mathcal{L}$.The spectral components $U_{1 L S \Omega_{1}}, \ldots, U_{N L S \Omega_{1}}$ represent the fundamental modes of vibrations of the image, and respectively describe increasing complexity of its geometric features, from coarse to fine scales.

Mapping the obtained spectra from the level set images decomposition on surfaces describe similar patterns of variations as the direct spectral decomposition of surfaces given by [9], as shown in Figure 3 .

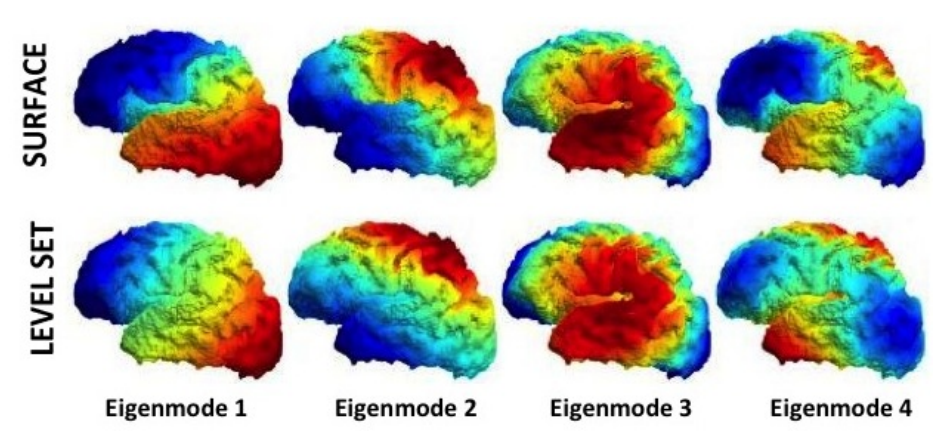

Fig. 1. Spectral modes of shape variation given by the decomposition of a subset of a level set image, and by the surface of the same boundary in the same subject 
Combined Level Set and Diffusion Tensor Spectra We combined the level set spectra with the spectra obtained by the decomposition of the diffusion tensor images as described by [4]. Briefly, for obtaining the DTI spectra, the weights between the graph nodes (also neighbouring voxels) are computed based on both tensor similarity from the log-Euclidean distance and Euclidean distance. Our main goal was to optimise the surface correspondence by taking into account microstrucural information inside the white matter. Hence, we separately compute tensor spectral components $U_{1 D T I \Omega_{2}}, \ldots, U_{N D T I \Omega_{2}}$ for a subset of the image $I_{D T I \Omega_{2}}$, in the deeper white matter structures, i.e. for the values inside the level set boundary (negative level set values) and outside the level set subset $I_{L S \Omega_{1}}$ $\left(I_{D T I \Omega_{2}} \cup I_{L S \Omega_{1}}=\varnothing\right)$. The independently computed spectra were then combined in the same space to obtain the combined spectra, with voxels receiving spectral information from either diffusion (inside the WM) or the surface data (around the boundary) $\left[U_{1 L S \Omega_{1}}, U_{1 D T I \Omega_{2}}\right], \ldots,\left[U_{N L S \Omega_{1}}, U_{N D T I \Omega_{2}}\right]$ (Figure 3 ).

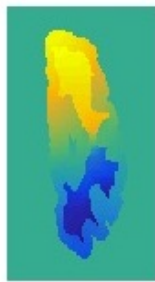

Spectra 1

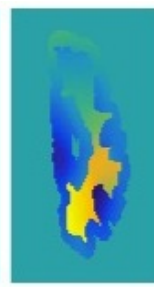

Spectra 2 Spectra 3

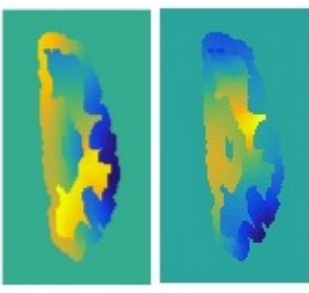

Spectra 4

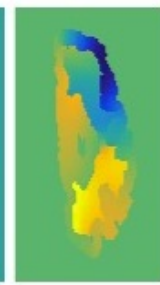

Spectra 5

Fig. 2. Combined spectral modes for the left hemisphere: shape variation given by the decomposition a subset of a level set image (edges of the surface) and microstructural variation given by the decomposition of the diffusion tensor image (inner)

Matching of multi-modal spectra Having the multi-modal spectra of two subjects $R$ and $F$, we can now estimate the spatial correspondences between them by optimising the correspondences between the spectral coordinates defined by the first $k$ multi-modal components of $\mathbf{U}^{\mathbf{R}}$, and $\mathbf{U}^{\mathbf{F}}$. We followed the computational scheme introduced in [9]. Briefly, the first $k$ spectral components are initially corrected for their sign ambiguity by computing the dot product between the corresponding eigenmodes at similar locations. For this we ran a coherent-point drift (CPD) rigid registration [10] of the respective clouds of points, which we used just to ensure the sign matching of the spectra in both the spectral and diffusion components, independently. Using the combined spectra and the thresholded level set distance maps for regularisation of the reference and floating images, we estimate a mapping between the corresponding points using a nearest neighbour search algorithm.

Comparison of Multi-Modal Spectral Matching with Surface Spectral Matching We used both PIMMS and JSM to create a groupwise average of the early time point of the subjects described in section 2 . The accuracy of the matching was evaluated by comparing at each vertex the standard deviation of 
the mean diffusivity values in the groupwise space. A lower variability indicates better alignment and consistency of the registration algorithm. We chose the $\mathrm{MD}$ for this validation over the FA, since FA values in the cortex are more homogeneous.

Figure 3 shows that the standard deviation from the mean of the mean diffusivity is minimised when we used the proposed multi-modal technique. Using additional microstructural information improves the alignment of the surfaces by taking into account tissue properties.
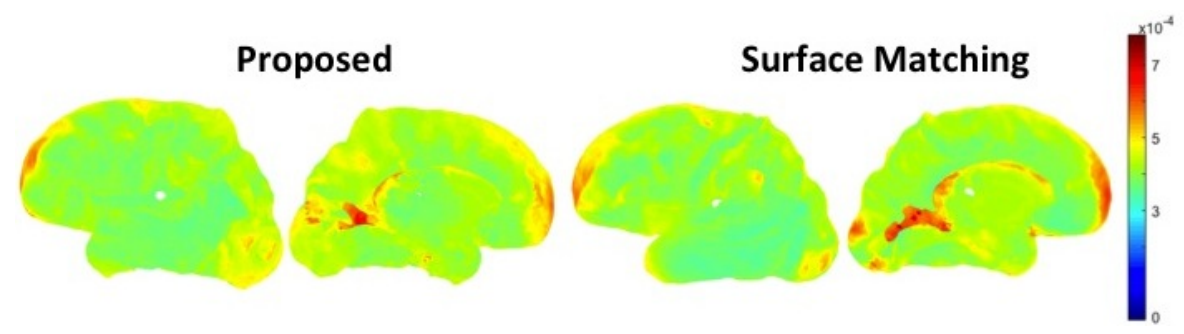

Fig. 3. Standard deviation in mean diffusivity in the cortex of the early timepoint on average groupwise for the left hemisphere, obtained using the proposed method and Joint-Spectral Matching of surfaces

\section{Groupwise Analysis of Longitudinal Changes}

Longitudinal Rates of Change All longitudinal changes in the parameters were corrected for the time between scans. We computed rates of change per week in CT, cortical FA and cortical MD during the preterm period in all infants and mapped them in the groupwise average space (Figure 4).

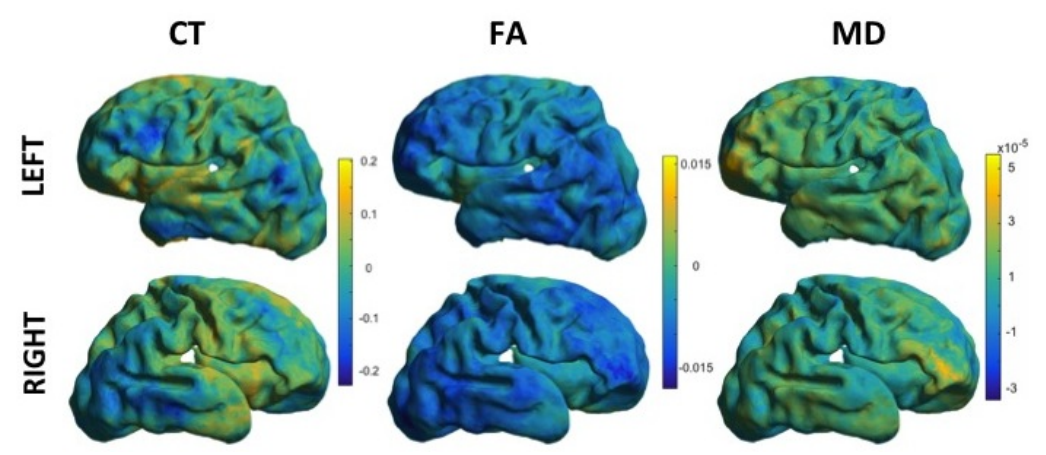

Fig. 4. Mean Longitudinal Rates of Change per week in cortical thickness (CT), cortical fractional anisotropy (FA) and cortical mean diffusivity (MD) in Groupwise Space 
Multi-modal parameters interdepencies To investigate correlations between the rates of change in the cortex, we computed the rank based correlation coefficient between rates in all infants, per region, for each pair of parameters: CT-FA, CT-MD and FA-MD. The p-values were FDR corrected at a 0.05 significance rate. The cortical rates of change show statistically significant correlations between the three measures in different regions summarised in Table 1. We notice that the changes in $\mathrm{CT}$ and FA are positively correlated, while the ones in CT-MD and FA-MD show a direct negative correlation.

Table 1. Statistically significant (0.05 significance, FDR corrected) correlation coefficients between multi-modal cortex parameters in all four lobes. Negative values imply a negative correlation, while positive imply a direct positive correlation. The regions not mentioned did not have a significant correlation between certain parameters.

\begin{tabular}{lcc}
\hline \hline & Left Hemisphere & Right Hemisphere \\
\hline CT-FA & Temporal: 0.15, Occipital: 0.09 & Temporal: 0.32, Occipital: 0.21 \\
CT-MD & Frontal: -0.06, Occipital: -0.13, & Frontal: -0.11 , Temporal: -0.33, \\
& Parietal: -0.17 & Occipital: -0.17 \\
FA-MD Frontal: -0.24, Temporal: -0.18, Parietal: -0.33 & - \\
\hline
\end{tabular}

\section{Discussion}

In this work we propose a novel registration technique based on Pairing Images using the Multi-Modal Spectra (PIMMS), which defines a surface-to-surface mapping in image domain by optimising both microstructural information in the white matter (from the diffusion tensor images) and WM/GM surface information (obtained from structural image segmentation). We applied this method to the challenging problem of registering early developmental stages in preterm born infants. Because of the timing, these surfaces do not provide us with sufficient sulcal patterns needed for a classical surface registration algorithm. The novelty of this method consists of ensuring a biologically accurate correspondence for surfaces with low gyrification.

We used PIMMS to create a groupwise average space of the early developmental time point, in which we mapped longitudinal changes over the preterm period in 9 infants. We investigated the rates of change per week in cortical thickness, cortical fractional anisotropy and cortical mean diffusivity in the cortex. The cortical FA is decreasing in most regions of the brain, and the cortical MD is increasing, results which match previous studies at the global level [6]. The cortical thickness is increasing in most regions, except the temporal lobe, where it is slightly decreasing. This result may be connected to the later development of the temporal lobe and the fact that it is the most affected region by preterm birth [5]. We further investigated the interdependency of these multi-modal parameters of the cortex across the different lobes. We found a positive CT-FA correlation, while the CT-MD and FA-MD correlations were negative. 
Our future research will imply linking grey and white matter properties close to the surface (e.g. studying cortical laminae in the cortex and closer to the white matter boundary), as well as linking the cortical surface and deep white matter connectivity. Furthermore this method may allow us to also look into the relationship between cortical folding and fibre-based connectivity.

Acknowledgements This work was supported by funding from the UK charity, SPARKS, EPSRC (EP/H046410/1, EP/J020990/1, EP/K005278), the MRC (MR/J01107X/1) and the National Institute for Health Research University College London Hospitals Biomedical Research Centre (NIHR BRC UCLH/UCL High Impact Initiative).

\section{References}

1. Marlow, N., Wolke, D., Bracewell, M.A., Samara, M.: Neurologic and Developmental Disability at Six Years of Age after Extremely Preterm Birth. New England Journal of Medicine 352(1) (2005) 9-19

2. Kapellou, O., Counsell, S.J., Kennea, N., Dyet, L., Saeed, N., Stark, J., Maalouf, E., Duggan, P., Ajayi-obe, M., Hajnal, J., Allsop, J.M., Boardman, J., Rutherford, M.A., Cowan, F., Edwards, A.D.: Abnormal cortical development after premature birth shown by altered allometric scaling of brain growth. PLoS medicine $3(8)$ (2006)

3. Wright, R., Makropoulos, A., Kyriakopoulou, V., Patkee, P., Koch, L., Rutherford, M., Hajnal, J., Rueckert, D., Aljabar, P.: Construction of a foetal spatio-temporal cortical surface atlas from in utero MRI: Application of spectral surface matching. NeuroImage 120 (2015) 467-480

4. Orasanu, E., Melbourne, A., Lorenzi, M., Modat, M., Eaton-rosen, Z., Robertson, N.J., Kendall, G., Ourselin, S.: Tensor Spectral Matching of Diffusion Weighted Images. In: SAMI Conference Proceedings, MIDAS Journal (2015) 35-44

5. Orasanu, E., Melbourne, A., Cardoso, M.J., Lomabert, H., Kendall, G.S., Robertson, N.J., Marlow, N., Ourselin, S.: Cortical folding of the preterm brain: a longitudinal analysis of extremely preterm born neonates using spectral matching. Brain and behavior 488 (2016) 1-18

6. Eaton-Rosen, Z., Melbourne, A., Orasanu, E., Cardoso, M.J., Modat, M., Bainbridge, A., Kendall, G.S., Robertson, N.J., Marlow, N., Ourselin, S.: Longitudinal measurement of the developing grey matter in preterm subjects using multi-modal MRI. NeuroImage 111 (2015) 580-589

7. Han, X., Pham, D.L., Tosun, D., Rettmann, M.E., Xu, C., Prince, J.L.: CRUISE: Cortical reconstruction using implicit surface evolution. NeuroImage 23 (2004) 997-1012

8. Waehnert, M.D., Dinse, J., Weiss, M., Streicher, M.N., Waehnert, P., Geyer, S., Turner, R., Bazin, P.: NeuroImage Anatomically motivated modeling of cortical laminae. NeuroImage 93 (2014) 210-220

9. Lombaert, H., Sporring, J., Siddiqi, K.: Diffeomorphic spectral matching of cortical surfaces. IPMI 7917 (2013) 376-389

10. Myronenko, A., Song, X.: Point set registration: coherent point drift. IEEE transactions on pattern analysis and machine intelligence 32(12) (dec 2010) 2262-75

11. Postelnicu, G., Zollei, L., Fischl, B.: Combined volumetric and surface registration. IEEE Trans Med Imaging 28(4) (2009) 508-522 